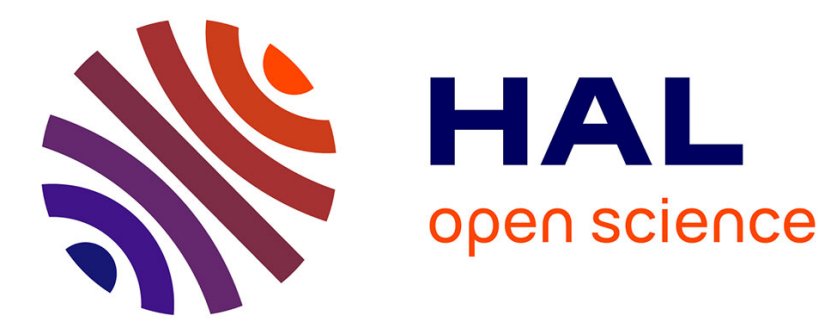

\title{
A Reliability Analysis of TSCH Protocol in a Mobile Scenario
}

\author{
Saleem Raza, Tim van Der Lee, George Exarchakos, Mesut Güneş
}

\section{To cite this version:}

Saleem Raza, Tim van Der Lee, George Exarchakos, Mesut Güneş. A Reliability Analysis of TSCH Protocol in a Mobile Scenario. 2019 16th IEEE Annual Consumer Communications \& Networking Conference (CCNC), Jan 2019, Las Vegas, France. pp.1-6, 10.1109/CCNC.2019.8651793 . hal-02915632

\section{HAL Id: hal-02915632 \\ https://hal.science/hal-02915632}

Submitted on 14 Aug 2020

HAL is a multi-disciplinary open access archive for the deposit and dissemination of scientific research documents, whether they are published or not. The documents may come from teaching and research institutions in France or abroad, or from public or private research centers.
L'archive ouverte pluridisciplinaire HAL, est destinée au dépôt et à la diffusion de documents scientifiques de niveau recherche, publiés ou non, émanant des établissements d'enseignement et de recherche français ou étrangers, des laboratoires publics ou privés. 


\title{
A Reliability Analysis of TSCH Protocol in a Mobile Scenario
}

\author{
Saleem Raza*, Tim van der Lee ${ }^{\dagger}$, George Exarchakos ${ }^{\dagger}$, and Mesut Güneş* \\ ${ }^{*}$ Communication and Networked Systems (ComSys), Faculty of Computer Science, \\ Otto-von-Guericke University, Universitätsplatz 2, 39106 Magdeburg, Germany \\ emails: $\{$ saleem.raza, mesut.guenes\}@ovgu.de \\ †Eindhoven University of Technology \\ P.O. Box 513, 5600MB Eindhoven, the Netherlands \\ emails: $\{$ t.lee, g.exarchakos\}etue.nl
}

\begin{abstract}
Future industrial low-power wireless networks together with cyber-physical systems demand mobile nodes to support diverse applications so as to increase operational efficiency and ensure autonomy. Mobility induces several challenges for low-power lossy networks due to dynamic topology, RF link instability, synchronization loss, signaling overhead, which lead to significant packet loss, more energy consumption, and higher latency. In this paper, we study a widely adopted time-slotted channel hopping (TSCH) MAC protocol in the presence of mobile nodes. We analyze how mobility impacts its reliability in terms of synchronization, message overhead, latency, and energy. The evaluation is performed through simulation and the results show that mobility may cause significant network downtime where nodes are unable to associate to the network for long period of time because of synchronization loss, especially if the environment is not fully covered by static nodes. Association and disassociation issues, induced by mobility, cause frequent disruptions in the network. Although, TSCH can handle mobility if the network space in which mobile nodes are evolving is fully covered by static nodes or there are enough mobile nodes to maintain a consistent coverage. However, the amount of message overhead to maintain synchronization is higher which impacts the reliability of the protocol in terms of energy and latency.

Index Terms-time-slotted channel hopping, medium access
\end{abstract} control, industrial low-power wireless networks, mobile nodes

\section{INTRODUCTION}

The growing adoption of industrial low-power wireless networks serves as the backbone for the low-power cyberphysical system (CPS). These low-power CPS are expected to encompass mobile nodes in near future. In this way, they promise to play a remarkable role for the automotive industry where mobile robots and equipment embedded with sensors and actuators can collaboratively exchange information to perform operations autonomously.

At the core of low-power CPS is the IEEE 802.15.4 [1] standard which is considered the de-facto physical (PHY) and medium access control (MAC) infrastructure for low-power wireless networks such as wireless sensor network (WSN), industrial wireless sensor and actuator network (IWSAN), etc. Time-slotted channel hopping (TSCH) [1] protocol is one of the MAC modes of IEEE 802.15.4 standard which overcomes the drawbacks [2] of less reliability and non-deterministic latency of the IEEE 802.15.4-2011 standard [3] MAC. It is

\footnotetext{
Note: The first two authors contributed equally to this work.
}

considered the most suitable protocol for the Internet of Things (IoT) in general and the industrial IoT in particular because of its low energy consumption and high reliability claims [4], [5]. Since, most of studies mainly undertake static nodes when using TSCH in several deployments, however, the evaluation of TSCH under mobility is an interesting case that takes into account wireless connectivity together with mobility to offer potential benefits.

In this paper, we study the performance of TSCH under node mobility. Particularly, we assess how mobility of nodes impacts network performance in terms of reliability, connection instability, signaling overhead/cost, and energy consumption. We define downtime as the main metric to see for how long nodes remain disconnected from the network and how reliability, latency, and energy are impacted. The evaluation analyzes the effect on synchronization due to node speed and the number of mobile nodes in the network. The results show that in order to decrease the downtime of network, the speed of mobile nodes need to be fast enough so that they remain connected. However, this increases message overhead which causes energy consumption and delay to increase and thus impacts the reliability of the protocol.

The remainder of the paper is organized as follows. We explain the technical overview of the TSCH protocol and the related work in Section II. Section III presents metrics and simulation set-up. We discuss results in Section IV and a conclusion is drawn in Section V.

\section{BACKGROUND AND RELATED WORK}

In this section, we provide a brief technical introduction of the TSCH protocol and we discuss two related studies pertaining to $\mathrm{TSCH}$ protocol under mobile nodes.

\section{A. TSCH MAC protocol}

TSCH is one of the MAC modes of the IEEE 802.15.4e2015 [1] standard which is an extended amendment to the original IEEE 802.15.4 [3] standard. It is considered the most suitable protocol particularly for harsh industrial deployments because it offers reliable and deterministic communication with the use of time-slotted access and channel hopping [4][6]. The time-slotted access efficiently addresses the lowpower requirement of the battery-operated nodes by enabling 

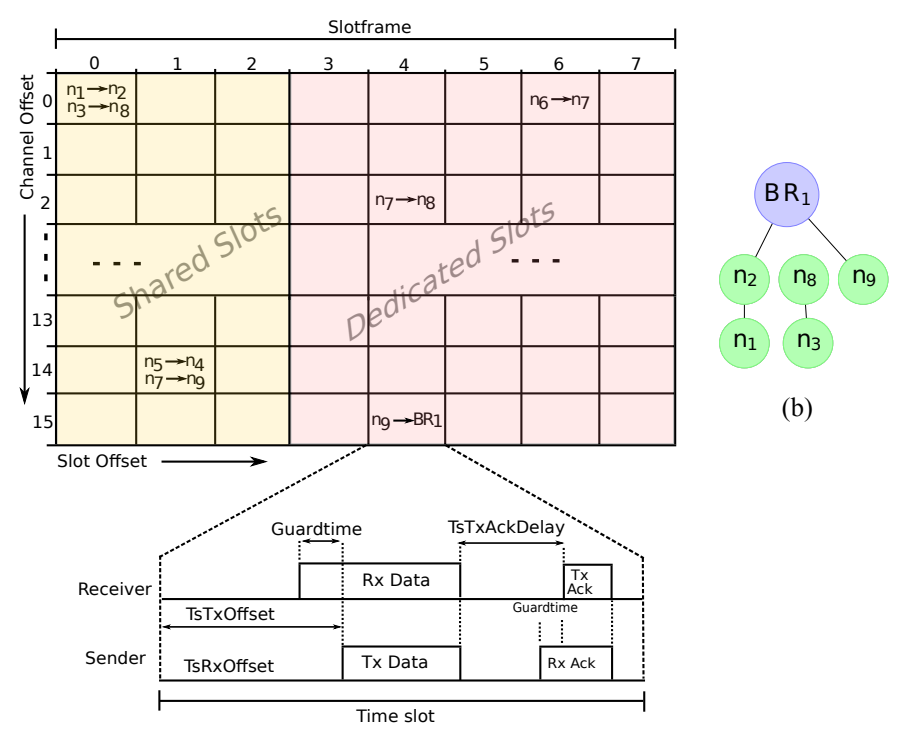

(a)

Figure 1. (a) TSCH slotframe showing dedicated and shared slots for the associated topology in (b). It also depicts typical sequence of events taking place within a slot between a sender and a receiver.

them to sleep/wakeup only when needed, whereas the channel hopping takes care of interference and fading of the wireless medium. In this way, it adequately satisfies the requirements of the constrained nodes and it can operate with other constrained protocols such as 6LoWPAN [7] or IPv6 routing protocol for low-power and lossy networks (RPL) [8].

TSCH provides guaranteed access for the medium to all the network nodes by allocating them a small portion of time called timeslot which is usually of $10 \mathrm{~ms}$ duration. The collection of multiple of timeslots is called a slotframe that repeats periodically as shown in Figure 1. The slotframe can be seen as a matrix of cells consisting of height equal to number of frequencies and width as the size of slotframe. Slotframe essentially coordinates a communication schedule (cells) which is a communication opportunity allocated to a sender-receiver node pair on an specific timeslot offset and channel offset. A frequency for a given cell can be computed through equation (1).

$$
\text { frequency }=F[(C O+A S N) \bmod n F r e q]
$$

Where Absolute Slot Number (ASN) acts as a time slot counter shared by all the devices. $A S N$ is initialized to 0 at the start of the network and is incremented globally in the network at every time slot [1]. $C O$ is channel offset, $F$ contains the set of available channels, $n$ Freq is the size of $F$. Channel offsets can be as many as there are available frequencies, even if the two nodes have the same channel offset, but a different $A S N$ will translate into a different frequency. However, to achieve reliable schedule, nodes need to be synchronized and require a mechanism to compensate for the anticipated clock drifting.

A slotframe can define dedicated timeslots as well as shared timeslots. Dedicated slots are contention-free which means that only a single sender-receiver node pair is allowed to communicate. The shared slots are contention-based where multiple sender-receiver node pairs are allowed to communicate and they are usually used for broadcast or signaling communication. As shown in Figure 1, a common practice is to locate shared slots at the beginning of the slotframe. For example, nodes $n_{1}$ and $n_{3}$ share a slot at channel 0 and timeslot 0 to forward their traffic to $n_{2}$ and $n_{8}$ respectively. It is possible that shared slots can encounter repeated collisions for which the standard defines a CSMA/CA mechanism to coordinate concurrent transmissions to reduce collision probability.

1) Network Advertising and Formation: The presence of TSCH network is advertised by the nodes through the transmission of enhanced beacons (EBs). An EB is a frame that contains information related to channel hopping, synchronization of timeslots, slotframe size, and the TSCH network in general. One of this information is the join-priority as defined by the standard [1]. Nodes looking to join the network continuously listen for EBs from other nodes and associate with network upon successful reception. When receiving several EBs, the preferred TSCH parent is chosen according to the join priority value. Capturing beacons is complex in TSCH networks due to channel hopping because nodes are not aware of the hopping pattern prior to joining the network. Therefore, they keep switching channel every fixed duration of time until an EB is received.

2) Synchronization: Synchronization of clocks is the fundamental requirement for TSCH to sustain a reliable communication. It requires clock synchronization accuracy in microseconds. Usually, the personal area network (PAN) coordinator or a border-router (BR) is the source of the clock synchronization for all the nodes and it initiates EB emission. In case the network does not have any PAN coordinator, the time-source is chosen as defined by the standard. Nodes in a TSCH network can disassociate from the network due to synchronization issues and thus need to resynchronize to their time-source. Such resynchronization takes place in two different ways: 1) frame-based 2) acknowledgement (ACK)-based. In the former, node upon successful reception of the frame adjusts its clock internally by comparing the frame reception time against the expected time. Whereas in the latter, the receiving node calculates the delta between the frame reception time against the frame expected time and embeds this information in the ACK back to the sender. Apart from this implicit synchronization during data exchange, TSCH also defines an additional way to maintain one-hop synchronization in the network which is based on the exchange of keep alive (KA) messages [9]. KA message are exchanged when node has not communicated to its neighbor for sometime because of having no traffic in the network and it is no more synchronized. Thus, node can exchange KA messages to resynchronize to the network.

\section{B. Related work}

In this section, we present related work that studied TSCH under node mobility. 
The impact of node mobility on TSCH protocol is studied in [10], where authors show how mobility causes disruptions in network and effects network performance. In this work, the focus is on comparison between TSCH and LLDN protocols. Particularly, the process of network association and disassociation incur signaling overhead for scanning the EBs which is costly for resynchronization attempts and energy. The signaling cost gets higher when multiple channels have to be scanned for longer time to rejoin the network causing not only more energy consumption but also increased latency. The evaluation is performed through simulation and authors reveal that synchronization issues can be mitigated by proper beacon and timeslot scheduling schemes. They argued that scanning on a single channel leads to low scanning time for network association thus saving on energy and latency.

The above work mainly undertakes the EBs scanning process into account which leads to more energy consumption and higher latency in the presence of mobile nodes. Whereas, our work focuses more on synchronization issues and we show that the mobile nodes do not consume more power and TSCH can handle mobility well if the space is fully covered by the mobile nodes.

The same authors have developed the mobility-aware framework in [11] called Mobile TSCH (MTSCH) which facilitates network association process for mobile nodes and reduces latency. The framework uses passive beacons instead of EBs to advertise the network. These passive beacons make use of ACK messages to be transmitted on a fixed channel by the network coordinator to advertise the network for other nodes. In this way, nodes only scan on a fixed channel rather scanning all available channels thus conserve energy and reduce latency to receive synchronization messages to join the network compared to default TSCH. The framework is evaluated through simulation and the results reveal that MTSCH is able to decrease the radio duty cycle of the mobile nodes by $7 \%$ to $50 \%$ on average and it increases network joining time of the nodes by $3 \%$ to $50 \%$.

\section{Simulation AND Experimental Setup}

In this section, we first describe the approach that explains the metrics considered. We present the experimental setup and the associated simulation environment.

\section{A. Approach}

We study the synchronization issues occurring in a mobile TSCH network. Especially, the issues pertain to frequent disassociation from the network due to mobility and attempting to resynchronize to the network so as to associate with it. In this regard, we analyze:

- Downtime: It is the percentage of time a node is out of the TSCH network. This is because mobile node cannot receive EBs, therefore cannot synchronize to the network and hence cannot communicate.

- Energy consumption: The energy consumption of the radio devices is calculated based on the assumption that a normal TSCH timeslot, either transmit or receive, consumes $12 \mathrm{~mA}$ in average and an idle slot consumes $0.4 \mathrm{~mA} \mathrm{[12]}$. Since only the synchronization process is considered, the energy consumption will increase if an application is using the TSCH network.

- Number of KA sent: The KA messages are unicast messages sent, besides EBs, to prevent the node to desynchronize from the network. The number of KA messages sent shows how close a node is to desynchronize. It is possible to incorporate the time information elements on data packet, thus reducing the number of KA messages when data is flowing through the network.

In case of an static network with all nodes inter-connected, the downtime is expected to be $0 \%$. However, mobile nodes resynchronize often with different neighbors. Even in a space fully covered with TSCH nodes, this resynchronization process sometimes makes the nodes disconnect and reconnect quickly thus causing the downtime to increase. Since a node with a high downtime can neither transmit nor receive any packet, this metric is critical and must be minimum to ensure a reliable performance of the network. We decided to test exclusively MAC-layer metrics and not application layer metrics because we noticed that the routing layer choice had the most impact on the application layer performance. However, these results are applicable to the application layer assuming no routing is used.

\section{B. Experimental Setup}

The evaluations are performed through simulation with the help of the Cooja emulator which is part of the ContikiOS [13], [14]. We generate the mobility for the nodes with the mobility framework ${ }^{1}$ available for Contiki-OS. Cooja emulates real nodes and guarantees the behavior and characteristics similar to reality.

A TSCH node requires to hear an EB from a neighbor node to be able to synchronize and join the network. To do so, all schedulers rely on a common shared slot among all nodes. To this extent, the IETF provides a minimal TSCH (TSCH_min) slotframe [7], which is a basic slotframe that defines an schedule having only one shared slot. In our experiments, we use the minimal TSCH slotframe to analyze the synchronization among all nodes. We configure the slotframe with the recommended parameters having the length of 11 timeslots and a timeslot duration of $10 \mathrm{~ms}$. The EB period is 16 seconds, meaning that a synchronized node emits an EB at least once every 16 seconds. Other schedulers may have different ways of initiating the synchronization process. For instance, the Orchestra [15] relies on the routing protocol RPL to schedule the EB slotframe. However, for RPL to know which neighbor nodes are in range, it relies on a slotframe identical to minimal $\mathrm{TSCH}$, with a size of 31 timeslots. Therefore, our analysis on the minimal slotframe does cover these cases. All the simulation parameters are summarized in Table I. We restrict the field size to $100 \times 100$ meter as it corresponds to the size of an industrial area where $\mathrm{TSCH}$ nodes are likely to

\footnotetext{
${ }^{1}$ http://anrg.usc.edu/contiki/index.php/Mobility_of_Nodes_in_Cooja
} 
Table I

SIMULATION PARAMETERS

\begin{tabular}{ll}
\hline Parameter & Value \\
\hline Field size & $100 \times 100 \mathrm{~m}$ \\
Transmission range & $100 \mathrm{~m}$ \\
Reception range & $50 \mathrm{~m}$ (with distance loss) \\
Network & Mobile \\
Mobility speed & 0 to $4 \mathrm{~m} \mathrm{~s}^{-1}$ \\
Mobility model & Random Walk \\
Number of nodes & Variable \\
Slotftrame length & 11 timeslots \\
Slot duration & $10 \mathrm{~ms}$ \\
Transceiver & CC2420 \\
Frequency spectrum & $2.4 \mathrm{GHz}$ \\
Propagation model & Two-ray model \\
\hline
\end{tabular}

be used. Here, a node covers $19.6 \%$ to $78.4 \%$ of the field in reception range depending on it's position. The transmission range of 1 node covers $78.4 \%$ to $100 \%$ of the field depending on its position. In our experiments, external perturbation are not considered, this means that the results obtained are the representative of the best-case (ideal) scenario. In all the experiments, the initial placement of the nodes is random. To gather accurate results, the tests are run between 10 and 30 times and the average metric is presented (in red), with the $95 \%$ confidence interval (CI) (in blue) to be discussed in Section IV. This means that $95 \%$ of all tests performed fall within this interval.

\section{Evalaution Results And Discussions}

In this section, we discuss the results based on the evaluation which takes into account the speed of the nodes, varying number of mobile nodes, and the space covered. In particular, we focus on the behavior and synchronization issues of a TSCH network by considering the above parameters.

\section{A. Speed}

First we analyze the effects of one mobile node's speed in a small three-nodes TSCH network. The mobile node follows a random walk model [16] in the restricted space of size $100 \times 100$ meter. In our model, we consider four possible steps (north, south, east, and west) each with the same distance $s$. One step is taken per second, therefore $s$ is also the speed in meter per second. In this experiment, the nodes cover a reception area from $20.31 \%$ to $99.39 \%$ of the field depending on their position. Transmission-wise, the coverage is comprised between $79.66 \%$ and $100 \%$ of the field. The results of this small proof of concept experiment are shown Figure 2. We can see in Figure 2a that the downtime is decreasing as the speed of the mobile node increases, and the $95 \%$ confidence interval is shrinking, meaning that the downtime is less impacted by the random initial placement of the nodes. In Figure $2 b$, we can see that the number of KA messages increases greatly as the speed of mobile nodes increases.

A mobile node with a speed close to 0 behaves similar to an static node. This means that if the mobile node is out of reach

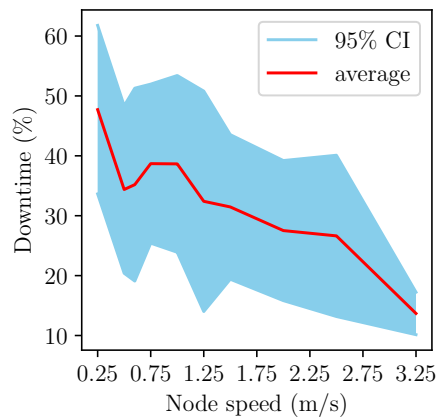

(a) Average downtime of all nodes in the network.

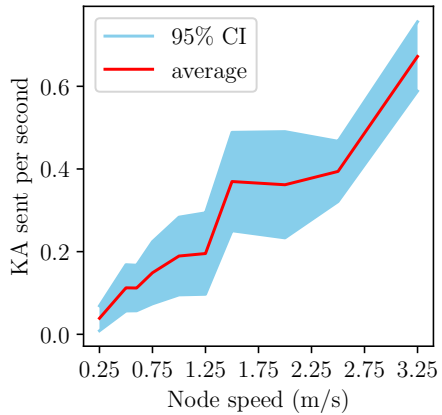

(b) Average number of KA messages sent per seconds.
Figure 2. Two static nodes and one mobile node with variable speed.

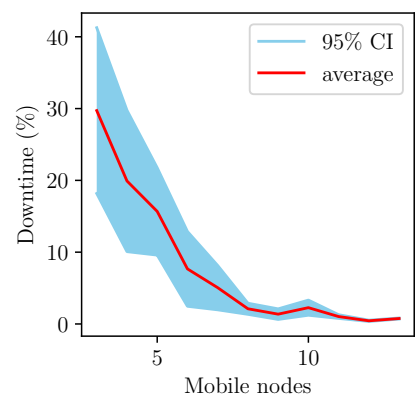

(a) Average downtime of all nodes in the network.

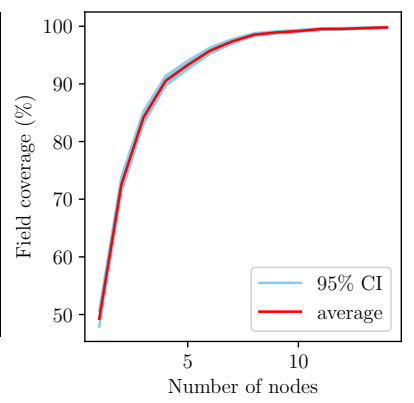

(b) Field coverage evaluation of the mobile nodes.
Figure 3. Downtime analysis when all nodes are mobile.

of the other nodes, it is not able to join the network quickly. On the contrary, if the mobile node has a very high speed, it is never out of the network which means it desynchronizes but resynchronizes very fast to the next parent node. This causes more KA messages to be sent to maintain synchronization which may impact the network performance by inducing delay and message overhead. This may not be the case if the time information element of TSCH is conveyed through data and if data is flowing through all nodes of the network.

\section{B. Number of Mobile Nodes}

In the second experiment, we analyze the behavior of the TSCH network when all nodes are mobile and the number of nodes increases. The speed of the nodes is set to $1 \mathrm{~m} / \mathrm{s}$. We analyze the downtime and the number of KA messages sent between 3 to 13 mobile nodes.

The results are presented in Figure 3 and Figure 4. From the downtime analysis, it appears that the average downtime and its standard deviation decrease when the number of mobile nodes increases as shown in Figure 3a. This can be related to the nodes' coverage area of the field, as shown in Figure $3 b$. Indeed, as all nodes move in a restricted space, increasing the number of mobile nodes also increases the coverage and thus the probability of each node to be in range of the network. In the $100 \times 100$ meter space, we see that after 10 nodes the downtime is minimum which is also the number of nodes for which we reach almost $100 \%$ coverage in average. 


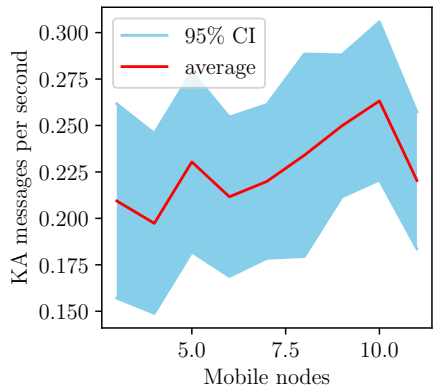

(a) Average number of KA sent per second and per node.

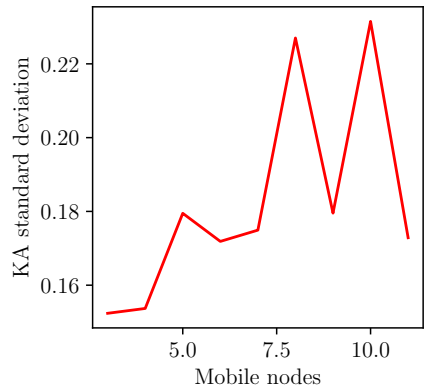

(b) Standard deviation of the average number of KA sent.
Figure 4. KA messages analysis when all nodes are mobile.

The KA messages analysis shows that a very low number of KA messages are sent per second and the standard deviation is also low as shown in Figure 4. More interestingly, it seems that these numbers are not impacted by the number of mobile nodes. Indeed, since all nodes have the same speed there is no difference if there is one or many mobile nodes, the network topology will change at the same speed. Since KA messages are sent to keep being synchronized with the TSCH parent, it is directly related to the network topology. Therefore, the required number of KA messages sent, to keep the network synchronized, stays the same regardless of the number of mobile nodes.

\section{In a Covered Space}

In this last experiment, we consider the field space of $100 \times 100$ meter which is covered by 4 nodes and they are placed in the coordinates $(30,30),(30,70),(70,70)$, and $(70,30)$ as depicted in Figure 5. With a transmission range of 50 meter, the entire space is covered by these 4 static nodes. This is typically the case of an industrial deployment where robots are moving and static gateways are ensuring stable communication. The number of mobile nodes varies from 1 to 3 nodes.

The results presented in Figure 6a and Figure $6 \mathrm{~b}$ show that regardless of the number of mobile nodes, the downtime is very low with less than $0.5 \%$ downtime. The average number of KA messages sent per second and per device is also very low within average 0.05 messages sent. This shows that in a fully covered environment, one or many nodes moving in this space will have no impact on the TSCH network performance and synchronization.

\section{Energy Consumption}

To evaluate if a mobile node is consuming more energy, the energy consumption of all nodes was analyzed in a scenario with 5 nodes. In this scenario, some nodes are mobile and some others are static. The number of mobile nodes varies from 0 to 5 . The results are depicted in Figure 7. It appears that there is no difference in energy consumption when the nodes are mobile or static.

Since the movement and placement of these nodes are random, at each time, an static node has the same probability

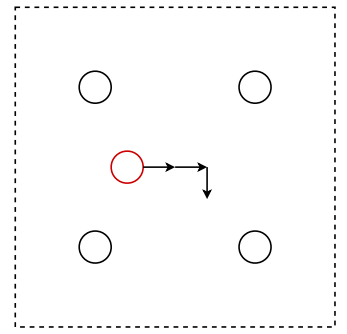

Figure 5. Representation of the covered space with one mobile node (in red) and four static nodes (in black).

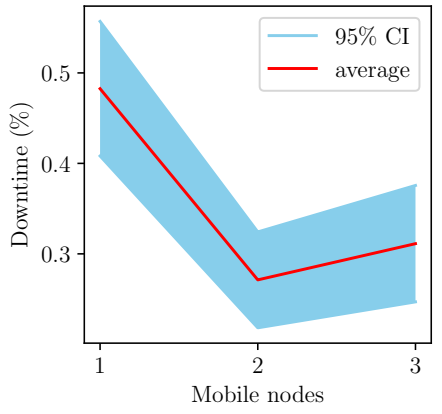

(a) Average downtime analysis.

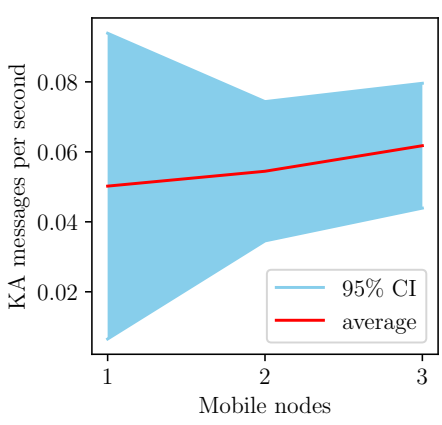

(b) KA messages analysis.
Figure 6. Result of the covered space experiment.

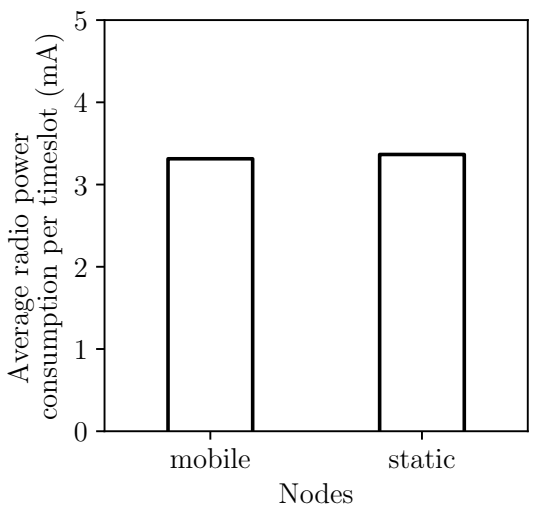

Figure 7. Energy consumption comparison in $m A$ per timeslot of mobile and static nodes.

of being disconnected from a mobile node than a mobile node being disconnected from an static node. Another way to see this, is to consider that from the point of view of mobile nodes, the static nodes are moving. Therefore, their energy consumption is similar with $3.3 \mathrm{~mA}$ per timeslot in average.

\section{SUMMARY}

This paper studies TSCH under node mobility. By analyzing the behavior of TSCH with variable node speed, it appears that having fast mobile nodes decreases the downtime of the network and ensures that all nodes can communicate to one an other. However, it significantly increases the number of KA messages sent and thus overloads the network, impacting energy and latency. When all nodes are mobile, the results show that once the space is fully covered, mobile nodes behave 
similarly to an static network; the downtime is close to 0 and the number of KA messages is stable, proportional to the speed of the nodes. Finally, we analyzed the behavior of TSCH in a fully covered space, a typical industrial deployment where mobile robots are evolving and static gateways are ensuring reliable communication. In this scenario, the number of mobile nodes shows no major impact on the network performance. Overall, the energy consumption of a mobile node is same as of an static node.

Our experimental results show that a TSCH network encounters little synchronization problems if the space where the mobile nodes are evolving is fully covered by static nodes, or if there are enough mobile nodes to guarantee consistent coverage. Therefore, using TSCH mobile nodes together with static nodes, which fully cover the environment, will ensure a healthy network behavior.

\section{ACKNOWLEDGMENT}

This research has been partially funded by the European Unions Horizon 2020 project INTER-IOT (grant number 687283).

\section{REFERENCES}

[1] IEEE standard for low-rate wireless networks. IEEE Std 802.15.4-2015 (Revision of IEEE Std 802.15.4-2011), pages 1-709, April 2016.

[2] Ali Nikoukar, Saleem Raza, Angelina Poole, Mesut Güneş, and Behnam Dezfouli. Low-power wireless for the internet of things: Standards and applications. IEEE Access (Early Access), 6(1):1-34, November 2018.

[3] IEEE standard for local and metropolitan area networks-Part 15.4: Lowrate wireless personal area networks (LR-WPANs). IEEE Std 802.15.42011 (Revision of IEEE Std 802.15.4-2006), pages 1-314, Sept 2011.

[4] I. Juc, O. Alphand, R. Guizzetti, M. Favre, and A. Duda. Energy consumption and performance of IEEE 802.15.4e TSCH and DSME. In IEEE Wireless Communications and Networking Conference, pages 1-7, April 2016.

[5] R. C. A. Alves and C. B. Margi. IEEE 802.15.4e TSCH mode performance analysis. In IEEE 13th International Conference on Mobile Ad Hoc and Sensor Systems (MASS), pages 361-362, Oct 2016.

[6] T. Watteyne, M. Palattella, and L. Grieco. Using IEEE 802.15.4e timeslotted channel hopping (TSCH) in the internet of things (iot): Problem statement. Internet Requests for Comments, May 2015. http://www. rfc-editor.org/rfc/rfc7554.txt.

[7] X. Vilajosana, K. Pister, and T. Watteyne. Minimal 6tisch configuration draft-ietf-6tisch-minimal-19. Internet Requests for Comments, 25, Jan. 2017, work in progress.

[8] T. Winter, P. Thubert, A. Brandt, J. Hui, R. Kelsey, P. Levis, K. Pister, R. Struik, JP. Vasseur, and R. Alexander. Rpl: Ipv6 routing protocol for low-power and lossy networks. Internet Requests for Comments, March 2012. http://www.rfc-editor.org/rfc/rfc6550.txt.

[9] Timothy Claeys. Initialisation of a wireless sensor network with the time. PhD thesis, Grenoble INP, 2015.

[10] Y. Al-Nidawi, H. Yahya, and A. H. Kemp. Impact of mobility on the iot mac infrastructure: IEEE 802.15.4e TSCH and LLDN platform. In 2015 IEEE 2nd World Forum on Internet of Things (WF-IoT), pages 478-483, Dec 2015.

[11] Y. Al-Nidawi and A. H. Kemp. Mobility aware framework for timeslotted channel hopping IEEE 802.15.4e sensor networks. IEEE Sensors Journal, 15(12):7112-7125, Dec 2015.

[12] I. Juc, O. Alphand, R. Guizzetti, M. Favre, and A. Duda. Energy consumption and performance of IEEE 802.15.4e TSCH and DSME. In IEEE Wireless Communications and Networking Conference, pages 1-7, April 2016.

[13] A. Dunkels, B. Gronvall, and T. Voigt. Contiki - a lightweight and flexible operating system for tiny networked sensors. In 29th Annual IEEE International Conference on Local Computer Networks, pages 455-462, Nov 2004.
[14] Contiki: The open source os for the internet of things. http://www. contiki-os.org/. Last visited - 20.04.2018.

[15] Simon Duquennoy, Beshr Al Nahas, Olaf Landsiedel, and Thomas Watteyne. Orchestra: Robust mesh networks through autonomously scheduled tsch. In Proceedings of the 13th ACM Conference on Embedded Networked Sensor Systems, SenSys '15, pages 337-350, New York, NY, USA, 2015. ACM.

[16] Tracy Camp, Jeff Boleng, and Vanessa Davies. A survey of mobility models for ad hoc network research. Wireless communications and mobile computing, 2(5):483-502, 2002. 